\title{
Chemical Properties of Element 105 in Aqueous Solution: Halide Complex Formation and Anion Exchange into Triisoctyl Amine
}

\author{
By J. V. Kratz, H. P. Zimmermann*, U. W. Scherer \\ Institut für Kernchemie, Universität Mainz, D-6500 Mainz, Federal Republic of Germany \\ M. Schädel, W. Brüchle \\ Gesellschaft für Schwerionenforschung mbH, D-6100 Darmstadt, Federal Republic of Germany \\ K. E. Gregorich, C. M. Gannett, H. L. Hall, R. A. Henderson, D. M. Lee, J. D. Leyba, M. J. Nurmia, \\ D. C. Hoffman, \\ Lawrence Berkeley Laboratory, University of California, Berkeley, CA 94720, USA \\ H. Gäggeler, D. Jost, U. Baltensperger, Ya Nai-Qi \\ Paul Scherrer Institut, CH-5232 Villigen, Switzerland
}

A. Türler and Ch. Lienert

Laboratorium für Radiochemie, Universität Bern, CH-3000 Bern 9, Switzerland

Dedicated to Prof. F. Baumgärtner on the occasion of his 60 th birthday

(Received March 14, 1989)

\section{Element 105 / Hahnium / Chemical properties / Group VB / Nuclear decay}

\begin{abstract}
Studies of the halide complexation of element 105 in aqueous solution were performed on $34-\mathrm{s}{ }^{262} \mathrm{Ha}$ produced in the ${ }^{249} \mathrm{Bk}\left({ }^{18} \mathrm{O}, 5 \mathrm{n}\right)$ reaction. The ${ }^{262} \mathrm{Ha}$ was detected by measuring the fission and alpha activities associated with its decay and the alpha decays of its daughter, $4.3-\mathrm{s}^{258} \mathrm{Lr}$. Time-correlated pairs of parent and daughter alpha particles provided a unique identification of the presence of ${ }^{262} \mathrm{Ha}$. About 1600 anion exchange separations of ${ }^{262} \mathrm{Ha}$ from $\mathrm{HCl}$ and mixed $\mathrm{HCl} / \mathrm{HF}$ solutions were performed on a one-minute time scale. Reversed-phase micro-chromatographic columns incorporating triisooctyl amine (TIOA) on an inert support were used in the computer-controlled liquid chromatography apparatus, ARCA II. ${ }^{262} \mathrm{Ha}$ was shown to be adsorbed on the column from either $12 \mathrm{M} \mathrm{HCl} / 0.02 \mathrm{M} \mathrm{HF}$ or $10 \mathrm{M} \mathrm{HCl}$ solutions like its homologs $\mathrm{Nb}$ and $\mathrm{Ta}$, and like $\mathrm{Pa}$. In elutions with $4 \mathrm{M} \mathrm{HCl} / 0.02 \mathrm{M} \mathrm{HF}$ (Pa-Nb fraction), and with $6 \mathrm{M} \mathrm{HNO}_{3} / 0.015 \mathrm{M} \mathrm{HF}$ (Ta fraction), the ${ }^{262} \mathrm{Ha}$ activity was found in the $\mathrm{Pa}-\mathrm{Nb}$ fraction showing that the anionic halide complexes are different from those of $\mathrm{Ta}$, and are more like those of $\mathrm{Nb}$ and $\mathrm{Pa}$. In separate elutions with $10 \mathrm{M} \mathrm{HCl} / 0.025 \mathrm{M} \mathrm{HF}$ (Pa fraction) and $6 \mathrm{M} \mathrm{HNO}_{3} / 0.015 \mathrm{M} \mathrm{HF}$ (stripping of $\mathrm{Nb}$ ) the ${ }^{262} \mathrm{Ha}$ was found to be equally divided between the $\mathrm{Pa}$ and $\mathrm{Nb}$ fractions. The non-tantalum like halide complexation of $\mathrm{Ha}$ is indicative of the formation of oxohalide or hydroxohalide complexes, like $\left[\mathrm{NbOCl}_{4}\right]^{-}$and $\left[\mathrm{PaOCl}_{4}\right]^{-}$or $\left[\mathrm{Pa}(\mathrm{OH})_{2} \mathrm{Cl}_{4}\right]^{-}$, at least for intermediate $\mathrm{HCl}$ concentrations, in contrast to the pure halide complexes in $\mathrm{Ta}$, like $\left[\mathrm{TaCl}_{6}\right]^{-}$.
\end{abstract}

\section{Introduction}

Previous chemical studies

The only studies of the chemistry of element 105 published so far were performed by Zvara et al. [1, 2]

\footnotetext{
* This work forms part of a doctoral thesis, Universität Mainz, to be submitted
}

and by Gregorich et al. [3]. The former used gas phase thermochromatography on the relatively volatile bromide and chloride compounds. They used the ${ }^{243} \mathrm{Am}$ $\left({ }^{22} \mathrm{Ne}, 4-5 \mathrm{n}\right)$ reaction to produce $2-\mathrm{s}{ }^{261-260} \mathrm{Ha}$ which they detected by spontaneous fission (SF) decay using track detectors along the thermochromatographic column. The observed position of fission tracks was similar to that of hafnium and was interpreted by Zvara et al. as being the expected position of ekatantalum. Quite different interpretations were suggested as well $[4,5]$. Also, it is not clear whether the SF activity observed was exclusively due to the decay of isotopes of element 105. Gregorich et al. [3] performed the first studies of the aqueous phase chemistry of element 105 (hahnium) on $34-\mathrm{s}{ }^{262} \mathrm{Ha}$ produced by the ${ }^{249} \mathrm{Bk}\left({ }^{18} \mathrm{O}, 5 \mathrm{n}\right)$ reaction. ${ }^{262} \mathrm{Ha}$ was uniquely identified by measuring the energy and time distribution of the alpha particles and fission fragments emitted in its decay. Time-correlated pairs of alpha particles from the decay of ${ }^{262} \mathrm{Ha}$ and its daughter, 4.3-s ${ }^{258} \mathrm{Lr}$, were also measured. Hahnium was found to adhere to glass surfaces upon fuming with concentrated nitric acid, a property very characteristic of the group-VB elements, niobium and tantalum, and of the pseudo-member of that group, protactinium. In other manually performed experiments, the extraction behaviour of hahnium fluoride complexes from mixed nitric acid/hydrofluoric acid solutions into methyl isobutyl ketone (MIBK) was investigated. The extraction of niobium and tantalum from acidic fluoride solutions into MIBK is well known, and has been used as a very characteristic separation and purification process for these elements [6-8]. Tantalum 
extracts under a broader range of conditions than does niobium. If this group-VB trend were to continue, hahnium should also form extractable species and be quantitatively extracted under the same conditions as tantalum is extracted. However, it was found that hahnium did not form extractable anionic fluoride complexes [3]. This non-tantalum like behavior was discussed [3] by extrapolation of group-VB chemical properties: The tendency to hydrolyze or form high coordination number fluoride complexes in hahnium may be much stronger than in tantalum, leading to non extractable species. It should be noted that the extraction conditions [3] were such that niobium was not extracted into the MIBK phase.

\section{Rationale for the present experiments}

The failure [3] to extract fluoride complexes of hahnium into MIBK under conditions in which tantalum is extracted quantitatively, presents a challenge. Perhaps the most obvious explanation is the formation of polynegative anions like $\left[\mathrm{HaF}_{7}\right]^{2-}$. The higher charge would then prevent extraction even into solvents with relatively high dielectric constants, such as MIBK. The existence of a different valence state, like $\mathrm{Ha}^{3+}$, with a $[\mathrm{Rn}] 5 f^{14} 7 s^{2}$ configuration due to the relativistic stabilization of the $7 s$ electrons was also discussed [3]. However, on the basis of the calculations of Desclaux [9] it was not considered likely except under very strongly reducing conditions.

While poly-negative species cannot be extracted into ketones it is possible to extract them by anion exchange into high molecular-weight ammonium salts. The successful extraction of hahnium into an amine phase would allow us to study the formation of anionic halide complexes of hahnium in more detail, and to compare them with the stabilities of halide complexes of its homologs $\mathrm{Nb}, \mathrm{Ta}$, and $\mathrm{Pa}$. A major focus in this work was to find conditions under which $\mathrm{Nb}, \mathrm{Ta}$, and $\mathrm{Pa}$ (and hopefully $\mathrm{Ha}$ ) would all be extracted simultaneously and quantitatively into a suitable amine, and to find other conditions for a selective back extraction of single homologs of hahnium into the aqueous phase. It would then be possible to study the distribution of ${ }^{262} \mathrm{Ha}$ activity among the $\mathrm{Nb}, \mathrm{Ta}$, and $\mathrm{Pa}$ fractions, and to learn about the similarity of hahnium chemistry to that of its homologs.

Amine extractions have another advantage for chemical studies of species that are produced as single atoms, such as ${ }^{262} \mathrm{Ha}$, so that their chemical behaviour must be studied on a "one-at-time" scale. Due to their high viscosity, high molecular weight ammonium salts are best suited as stationary phase on inert column support materials for high performance liquid chromatography (HPLC). Then, the principle of chromatography can be applied to single atoms or ions, and the many adsorption-desorption cycles along the column in the elution of that ion ensure a statistical chemical behaviour, so that one can be reasonably
Table 1. Alpha-decay energies of ${ }^{262} \mathrm{Ha}$ and ${ }^{258} \mathrm{Lr}$ [16]

\begin{tabular}{|c|c|c|c|}
\hline \multicolumn{2}{|c|}{${ }^{262} \mathrm{Ha}\left(t_{1 / 2}=34 \pm 4 \mathrm{~s}\right)$} & \multicolumn{2}{|c|}{${ }^{258} \operatorname{Lr}\left(t_{1 / 2}=4.3 \pm 0.5 \mathrm{~s}\right)$} \\
\hline Energy (keV) & Abundance & Energy $(\mathrm{keV})$ & Abundance \\
\hline 8450 & $75 \%$ & 8565 & $20 \%$ \\
\hline 8530 & $16 \%$ & 8595 & $46 \%$ \\
\hline \multirow[t]{2}{*}{8670} & $9 \%$ & 8621 & $25 \%$ \\
\hline & & 8654 & $9 \%$ \\
\hline
\end{tabular}

certain that the observations represent the "true" chemistry of the elements.

Furthermore, HPLC techniques lend themselves easily to automation. Because of the short half-life and the low production rate (enough for the detection of a few atoms per hour) of ${ }^{262} \mathrm{Ha}$, rapid, automatic separations that could be repeated on a one-minute time scale over many hours of operation were developed and realized by the miniaturized Automatic Rapid Chemistry Apparatus, ARCA II, described in detail in a companion paper [10].

\section{Nuclear decay properties of ${ }^{262} \mathrm{Ha}$}

The decay of $34-\mathrm{s}{ }^{262} \mathrm{Ha}$ has been studied by a number of groups [3,11-15]. A summary of the alpha-decay energies and abundances [16] is given in Table 1. According to the original data by Ghiorso et al. [11] and the present data, the $8.53 \mathrm{MeV}$ branch in ${ }^{262} \mathrm{Ha}$ is probably not as abundant as given in Table 1 , at least its intensity is not larger than that of the $8.67 \mathrm{MeV}$ group.

A SF activity with a half-life consistent with the alpha-decay half-life of ${ }^{262} \mathrm{Ha}$ was also observed [1115]. According to Gregorich [15], in the ${ }^{18} \mathrm{O}+{ }^{249} \mathrm{Bk}$ reaction at $102 \mathrm{MeV}$, the fission cross section is $4.3 \pm 1.0 \mathrm{nb}$, the alpha cross section is $1.4 \pm 0.5 \mathrm{nb}$, so that a fission branch of $75 \pm 10 \%$ results. It is not clear whether this is a true fission branch in ${ }^{262} \mathrm{Ha}$, or due to the SF decay of its electron-capture daughter, 60 $\mathrm{ms}{ }^{262} \mathrm{Rf}$. In the recent chemical studies on ${ }^{262} \mathrm{Ha}$ [3], the fission branch was found to be smaller, $49 \pm 13 \%$. This discrepancy between the previous, purely instrumental measurements, and the chemical experiments may best be explained by postulating another SF activity with a similar half-life, not due to the decay of a hahnium isotope, but to the decay of another actinide or transactinide nuclide, which is produced in the ${ }^{18} \mathrm{O}+{ }^{249} \mathrm{Bk}$ reaction. This demonstrates the unspecific nature of the SF decay mode for the assignment of $\mathrm{A}$ and $\mathrm{Z}$.

The more specific method for detecting ${ }^{262} \mathrm{Ha}[3$, $11,15]$ is the detection of alpha particles from the decay of ${ }^{262} \mathrm{Ha}$, and/or its daughter, ${ }^{258} \mathrm{Lr}$. Both alpha-particle energies and decay times can then be used as identification criteria. With sufficient chemical decontamination from competing activities, these criteria provide an unambiguous identification. Even 
more unique is the detection of time-correlated pairs of parent and daughter alpha particles [11, 3], i.e. the detection of an alpha particle of ${ }^{262} \mathrm{Ha}$ followed closely in time by an alpha particle from its 4.3-s daughter, ${ }^{258} \mathrm{Lr}$.

\section{Distribution of $\mathrm{Zr}, \mathrm{Hf}, \mathrm{Nb}, \mathrm{Ta}$, and $\mathrm{Pa}$ between mineral acids and aliphatic amines}

\section{Batch extraction experiments}

In order to survey systematically the extraction behaviour of group-IVB and -VB elements with aliphatic amines we have performed batch extractions in a number of mineral acid/amine systems [17]. Additional information can, of course, be obtained from the literature, see for example Ref. [18]. The following amines were used and are listed with the manufacturer: triisoctyl amine (TIOA), Fluka; trioctyl amine (TOA), Merck; Alamine 336 (tricaprylyl amine), General Mills; and methyl-tricaprylyl ammonium chloride (MTCA•Cl), ICN K\&K. Mineral acids were $\mathrm{H}_{2} \mathrm{SO}_{4}$, $\mathrm{HNO}_{3}, \mathrm{HCl}, \mathrm{HF}$, mixed $\mathrm{HCl} / \mathrm{HF}$, and mixed $\mathrm{HNO}_{3} /$ HF. The amines were sorbed on sieved Voltalef ${ }^{R} 300$ LD-CHR (polytrifluoromonochloro ethylene, Lehmann \& Voss, Hamburg) with a particle size of $125-$ $250 \mu \mathrm{m}$. The weight ratio of amine to support was $1: 2$. Prior to the extraction experiments the amine/ Voltalef $^{\mathrm{R}}$ powder was converted to the respective ammonium salt by shaking with a $2 \mathrm{M}$ solution of the mineral acid. The powder was then rinsed with water and dried. Batches of $0.5 \mathrm{~g}$ of the powder and $2 \mathrm{ml}$ of a mineral acid solution of given molarity containing radioactive tracers of $\mathrm{Zr}, \mathrm{Hf}, \mathrm{Nb}, \mathrm{Ta}$, and sometimes $\mathrm{Pa}$, were contacted in polyethylene flasks by shaking for $15 \mathrm{~min}$. The phases were conveniently separated by filtration and an aliquot of the aqueous phase was assayed by $\gamma$-ray spectroscopy in order to determine the retention of the tracers in the aqueous phase. The tracers were stored in hydrofluoric acid solution to prevent hydrolysis. Shortly before the extraction experiment, the fluoride ion in the tracer solution was removed by addition of an excess of $\mathrm{H}_{3} \mathrm{BO}_{3}$. Fig. 1 shows typical results for TIOA which was finally selected for the element 105 experiments because of certain advantages over the other amines.

Generally, in amine/mineral acid systems, there is a competition between the extraction of anionic metal complex and the extraction of the acid. The latter is predominant in hydrofluoric acid solutions because of the high degree of covalent bonding in $\operatorname{HF}[19,20]$ :

$$
\left[\mathrm{R}_{3} \mathrm{NH}^{+} \mathrm{F}^{-}\right]+\mathrm{HF} \rightleftharpoons\left[\mathrm{R}_{3} \mathrm{NH}^{+} \mathrm{HF}_{2}^{-}\right]
$$

The extraction of the metal species can be described in two ways [19]:

i) as an ion-exchange mechanism, i.e. anionic metal complexes are exchanged against the mineral acid anion according to

$$
\begin{aligned}
& m\left[\mathrm{R}_{3} \mathrm{NH}^{+} \mathrm{X}^{-}\right]+\left[\mathrm{MX}_{m+n}\right]^{m-} \\
& \rightleftharpoons\left[\left(\mathrm{R}_{3} \mathrm{NH}^{+}\right)_{m}\left(\mathrm{MX}_{m+n}\right)^{m-}\right]+m \mathrm{X}^{-},
\end{aligned}
$$

and

ii) as formation of ion pairs, i.e. neutral metal complexes form adducts with the ammonium salt according to

$$
\begin{aligned}
& m\left[\mathrm{R}_{3} \mathrm{NH}^{+} \mathrm{X}^{-}\right]+\mathrm{MX}_{n} \\
& \rightleftharpoons\left[\left(\mathrm{R}_{3} \mathrm{NH}^{+}\right)_{m}\left(\mathrm{MX}_{m+n}\right)^{m-}\right] .
\end{aligned}
$$

Which description is to be preferred depends on the conditions in the aqueous phase. At low acid concentrations there is preferential formation of adducts, eq. (3). With increasing acid molarity the concurrent acid extraction displaces the neutral metal complexes. At higher acid concentrations the formation of anionic metal complexes becomes increasingly important, and these are extracted preferentially into the organic phase. The details of these concurrent processes depend on the dissociation constant of the acid, the complex formation constants for various competing forms of complexes (varying coordination number), on the nature of the ligand, etc. The ups and downs in the extraction yields in Fig. 1, most pronounced for $\mathrm{Nb}$ species in the TIOA/HCl, TIOA/HCl/HF, and to some extent in the $\mathrm{TIOA} / \mathrm{H}_{2} \mathrm{SO}_{4}$ system, indicate that there is indeed a competition among several equilibria such as the ones given by eq. (1), (2), and (3).

Our major focus for the element 105 separations was to find conditions under which the group-VB elements are simultaneously extracted into the amine while the possibly interfering group-IVB elements are not. This condition is fulfilled for TIOA, see Fig. 1, in $12 \mathrm{M} \mathrm{HF}, 10 \mathrm{M} \mathrm{HCl}$, and $12 \mathrm{M} \mathrm{HCl} / 0.03 \mathrm{M} \mathrm{HF}$. We have selected $10 \mathrm{M} \mathrm{HCl}$ and $12 \mathrm{M} \mathrm{HCl} / 0.02 \mathrm{M} \mathrm{HF}$ for the hahnium experiments because working with $12 \mathrm{M} \mathrm{HF}$ is not very attractive. Also, it was found that a back extraction of Ta from a HF loaded amine phase takes large volumes of effluent, and therefore takes too much time for the required fast separations. A comparison of Figs. $1 \mathrm{~b}$ and $1 \mathrm{e}$, for example, demonstrates the role of HF extraction, eq. (1), even if the $\mathrm{HF}$ concentration is rather low: In pure $\mathrm{HCl}, \mathrm{Zr}$ and $\mathrm{Hf}$ are extracted into the amine in $12 \mathrm{M} \mathrm{HCl}$; in $12 \mathrm{M}$ $\mathrm{HCl} / 0.03 \mathrm{M} \mathrm{HF}$ they are not; in $10 \mathrm{M} \mathrm{HCl}, \mathrm{Nb}$ is already quantitatively extracted; in the presence of $0.03 \mathrm{M} \mathrm{HF}$ quantitative extraction requires an increase of the $\mathrm{HCl}$ concentration to $12 \mathrm{M} \mathrm{HCl}$. The latter cannot easily be seen in Fig. 1e, which is due to the logarithmic scale of the $\mathrm{HCl}$ molarity. We have made use of these subtle differences in the column separations, e.g., in a separation of $\mathrm{Pa}$ from $\mathrm{Nb}$ in $10 \mathrm{M} \mathrm{HCl} / 0.025 \mathrm{M} \mathrm{HF}$, see below. A possibility for a very good separation of $\mathrm{Pa}$ and $\mathrm{Nb}$ from $\mathrm{Ta}$ is their elution (Fig. 1e) from the column in $4 \mathrm{M} \mathrm{HCl} / 0.03 \mathrm{M}$ $\mathrm{HF}$. The most efficient back extraction of Ta from the amine was found to occur in $6 \mathrm{M} \mathrm{HNO}_{3} / 0.03 \mathrm{M} \mathrm{HF}$, see Fig. 1f. There might be other possibilities for achieving back extraction of Ta, e.g., into dilute $\mathrm{HCl}$ (Fig. 1b) or dilute HF (Fig. 1d). 
The extraction data with TOA are very similar to those in Fig. 1. Because the back extraction of Ta with $6 \mathrm{M} \mathrm{HNO}_{3} / 0.03 \mathrm{M} \mathrm{HF}$ is less efficient with TOA than with TIOA, we have decided against the use of TOA. This is also the main reason for not using $\mathrm{MTCA} \bullet \mathrm{Cl}$, where a back extraction of Ta into the aqueous phase is nearly impossible, or Alamine 336, where, again, there are no good prospects for back extracting Ta. In the latter systems the conditions for the proposed separations are also less favorable due to a stronger extraction of the group-IVB elements in strong hydrochloric acid.

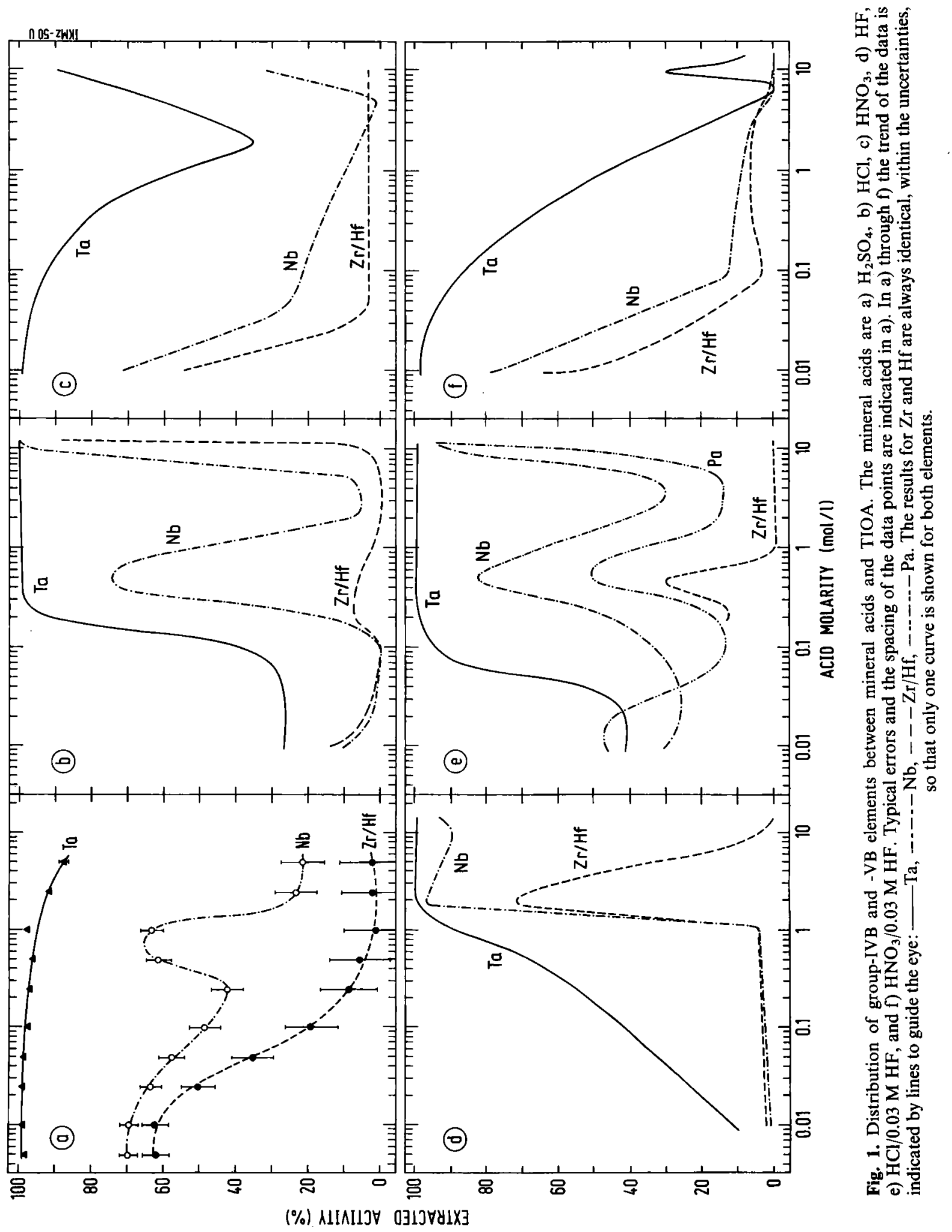




\section{HPLC column separations}

As a next step, the favorable conditions for a common extraction of $\mathrm{Nb}, \mathrm{Ta}$, and $\mathrm{Pa}$ into TIOA $(10 \mathrm{M} \mathrm{HCl}$, and $12 \mathrm{M} \mathrm{HCl} / 0.03 \mathrm{M} \mathrm{HF}$ ) and possibilities for their specific elution from the amine, visible in Fig. 1, were tested in manually performed HPLC separations. Voltalef ${ }^{\mathrm{R}}$ powder as inert support coated with TIOA was used as stationary phase and packed under pressure into Teflon columns of $1.7 \mathrm{~mm}$ inner diameter and variable height from a slurry in $4 \mathrm{M} \mathrm{HCl}$. The whole system, including the pump (LATEK, Heidelberg) consisted of Teflon, Kel-F, and sapphire, because it was found that any contact of the tracer activities with glass (or with silica-gel type column support materials) lead inevitably to yield losses due to adsorption at the surfaces. Radioactive tracers were introduced through a sample loop. Parameters that were optimized in these separations were the weight ratio between TIOA and Voltalef ${ }^{\mathrm{R}}$, the grain size of the sieved Voltalef ${ }^{R}$, the column height, flow rate, and fraction size. Also, there was some fine tuning of the HF concentrations. The following conditions were found to be optimum, or tolerable compromises, between a high quality separation and the required speed: Column size $1.7 \times 11 \mathrm{~mm}$, filled with Voltalef $(10-25 \mu \mathrm{m})$, weight ratio, amine to support, 1:5. The columns were run at room temperature to facilitate their rapid replacement with new ones in the hahnium experiments. The flow rate was $1.4 \mathrm{ml} / \mathrm{min}$. A separation of $\mathrm{Zr}, \mathrm{Nb}-\mathrm{Pa}$, and $\mathrm{Ta}$ under these conditions is shown in a companion paper [10]. Eu, which was used as a prototype for +3 ions, i.e., for the heavy actinides, was found to elute exactly with $\mathrm{Zr}$. $\mathrm{Nb}$, $\mathrm{Ta}$, and $\mathrm{Pa}$ were extracted from $12 \mathrm{M} \mathrm{HCl} / 0.02 \mathrm{M} \mathrm{HF}$ while $\mathrm{Zr}$ (and $\mathrm{Eu}$ ) were eluted with the feeding solution, as suggested by Fig. 1e. Then, $\mathrm{Nb}$ and $\mathrm{Pa}$ were eluted together with $4 \mathrm{M} \mathrm{HCl} / 0.02 \mathrm{M} \mathrm{HF}$, and, finally, Ta was stripped from the column in $6 \mathrm{M} \mathrm{HNO}_{3} / 0.015 \mathrm{M}$ HF. These, and similar conditions served as a basis for the design and construction of ARCA II.

\section{Automated separations with ARCA II}

ARCA II [10] consisted of three chemically inert HPLC pumps, each pumping one eluent, e.g., one $12 \mathrm{M} \mathrm{HCl} / 0.02 \mathrm{M} \mathrm{HF}$, the other $4 \mathrm{M} \mathrm{HCl} / 0.02 \mathrm{M} \mathrm{HF}$, and the third $6 \mathrm{M} \mathrm{HNO}_{3} / 0.015 \mathrm{M} \mathrm{HF}$, through Teflon tubing of $0.3 \mathrm{~mm}$ i.d. to the central Teflon unit containing various Kel-F sliders and two movable magazines containing twenty chromatographic columns $(1.6 \times 8 \mathrm{~mm})$ each. The column material was TIOA/ Voltalef $^{R}(32-63 \mu \mathrm{m})$, weight ratio 1:5. The larger particle size and a somewhat reduced flow rate $(1.0 \mathrm{ml} /$ min), as compared to the manual HPLC separations, was selected in order to keep the pressure below 10 bar which was necessary to prevent the system from leaking. The $\mathrm{He} / \mathrm{KCl}$-gas jet deposited the transported reaction products continuously onto one of two alternating polyethylene frits of $35 \mu \mathrm{m}$ pore size. After a one minute collection, the first frit was moved on top of one of the mini columns, washed with $12 \mathrm{M} \mathrm{HCl}$ / $0.02 \mathrm{M} \mathrm{HF}$, whereby the reaction products were dissolved, complexed, and extracted into the amine (in case of $\mathrm{Nb}, \mathrm{Ta}$, and $\mathrm{Pa}$ ), while the non-extractable species ran through into the waste $(\approx 10 \mathrm{~s})$. The column was then washed with $4 \mathrm{M} \mathrm{HCl} / 0.02 \mathrm{M} \mathrm{HF}$ for about ten seconds and the effluent (containing $\mathrm{Nb}$ and $\mathrm{Pa}$ ) was collected on a Ta disc and quickly evaporated to dryness by intense infra-red light and hot He gas. Next, the Ta fraction was eluted in ten seconds with $6 \mathrm{M} \mathrm{HNO}_{3} / 0.015 \mathrm{M} \mathrm{HF}$, collected on a Ta disc, and evaporated to dryness. The Ta discs were flamed, cooled to room temperature and inserted into the counting chambers about $55 \mathrm{~s}$ after the end of collection. Five seconds later the next one-minute collection of activity on the twin frit was complete. That frit was moved on top of another mini column contained in the opposite magazine and the next separation cycle was carried out. After each separation, the magazines were moved by one step, thus introducing a new mini column into the elution position. In this way the time consuming reconditioning of used columns was avoided. After 40 minutes of continuous collection and separation cycles the program was stopped, the used magazines were removed, and two new magazines were introduced, and another 40 cycles were started. Typically, 300 experiments were carried out in one shift of beam time ( 8 hours). The time sequences were optimized in test runs with radioactive tracers of $\mathrm{Zr}, \mathrm{Nb}, \mathrm{Hf}, \mathrm{Ta}$, and $\mathrm{Pa}$. Also, on-line tests of the performance of ARCA II were conducted with short-lived fission products at the Mainz TRIGA reactor.

\section{Production, transport, and detection of ${ }^{262} \mathrm{Ha}$}

\section{Production and transport}

${ }^{262} \mathrm{Ha}$ was produced at the LBL 88 -inch Cyclotron by the ${ }^{249} \mathrm{Bk}\left({ }^{18} \mathrm{O}, 5 \mathrm{n}\right)$ reaction. A $114 \mathrm{MeV}{ }^{18} \mathrm{O}^{5+}$ beam passed through a $1.8 \mathrm{mg} / \mathrm{cm}^{2}$ HAVAR window, $0.2 \mathrm{mg} / \mathrm{cm}^{2}$ of $\mathrm{N}_{2}$ cooling gas, and the $2.46 \mathrm{mg} / \mathrm{cm}^{2}$ Be target backing before passing through the ${ }^{249} \mathrm{Bk}$ target material. The energy in the target was $98-$ $99 \mathrm{MeV}$. The target originally consisted of $995 \mu \mathrm{g} / \mathrm{cm}^{2}$ of freshly purified ${ }^{249} \mathrm{Bk}$ deposited on the Be backing by molecular plating and converted to the oxide. $\mathrm{Du}$ ring the experiments the target composition varied between 0.932 and $0.895 \mathrm{mg} / \mathrm{cm}^{2}$ of $330-\mathrm{d}^{249} \mathrm{Bk}$ due to its decay, with the remainder of the total thickness being its ${ }^{249} \mathrm{Cf}$ daughter. The beam currents varied between 0.4 and 0.5 particle microamperes. Under these conditions, the production rate for alpha-decaying ${ }^{262} \mathrm{Ha}$ was sufficient to give about one alpha decay at the end of a one-minute irradiation.

The reaction products recoiling out of the target were stopped in $\mathrm{He}$ gas at 1.2 bar which had been loaded with $\mathrm{KCl}$ aerosols. The activity, attached to the aerosols, was swept out of the recoil chamber with 
the gas into a polyvinyl chloride capillary $(1.34 \mathrm{~mm}$ i.d.) and transported $5 \mathrm{~m}$ to the collection site in ARCA II. There, the transport gas was pumped through a polyethylene frit by a mechanical pump. The activity-bearing aerosols were collected on that frit, and, after the end of the collection time, the frit was shuttled into the position for chemical processing of the activity, as described in Section 2.

$\mathrm{The} \mathrm{He} / \mathrm{KCl}$-jet transport efficiency was measured several times during the experiments. This was done by dissolving the activity in $12 \mathrm{M} \mathrm{HCl} / 0.02 \mathrm{M} \mathrm{HF}$ and eluting it through an empty column directly onto a $\mathrm{Ta}$ disc. After careful evaporation to dryness and flaming, the production rate of the ${ }^{252-255} \mathrm{Fm}$ transfer products was determined by pulse-height analysis. At the beginning and at the end of the hahnium experiments, separate bombardments of the Bk target were performed in which all of the products recoiling from the target were caught in a gold catcher foil located directly behind the target. After one hour of irradiation the foil was dissolved in concentrated $\mathrm{HCl}$ $\left(\mathrm{HNO}_{3}\right)$ to which an aliquot of ${ }^{241} \mathrm{Am}$ tracer had been added to trace the chemical yield of actinides. The gold was removed on an anion exchange column. The actinide fraction, which passed through the column, was collected and dried on a Pt disc for alpha spectroscopy. By comparing the apparent production rates measured after transport through the $\mathrm{He} / \mathrm{KCl}$ jet with the absolute production rates from these direct catcher foil experiments, the jet efficiency was determined. The jet transport efficiency was found to vary between 34 and $52 \%$ during the production runs.

\section{Production of tracers for on-line chemical studies}

Because it was desirable to study the chemical properties of $\mathrm{Zr}, \mathrm{Nb}, \mathrm{Hf}, \mathrm{Ta}$, and $\mathrm{Pa}$ and of some lanthanides, under the same conditions as used for the hahnium experiments, carrier-free activities of these elements were freshly produced on-line and subjected to the same chemical procedures in ARCA II. These were produced in $\left({ }^{4} \mathrm{He}, \mathrm{xn}\right)$ reactions with an $80 \mathrm{MeV}{ }^{4} \mathrm{He}$ beam on strontium chloride, and yttrium, praseodymium, ytterbium and lutetium metal targets, respectively. Because of the smaller recoil range in fusion reactions with alpha particles, the gas-jet system parameters were changed for these tracer experiments. In other experiments, $32-\mathrm{s}{ }^{166} \mathrm{Ta}$ was produced in the ${ }^{\text {nat }} \mathrm{Eu}\left({ }^{20} \mathrm{Ne}, 5-7 \mathrm{n}\right)$ reaction using a $118 \mathrm{MeV}$ (on target) ${ }^{20} \mathrm{Ne}$ beam. This activity was transported to the collection frit under exactly the same conditions as used for the hahnium experiments.

The effluents from the TIOA mini columns were collected in fractions of typically three drops $(\approx 10 \mu \mathrm{l}$ per drop) in small test tubes and assayed for $\gamma$-ray activities using two $\mathrm{Ge}$ detectors. In agreement with earlier tracer studies, chemical yields for $\mathrm{Nb}, \mathrm{Ta}$, and $\mathrm{Pa}$ were found to be consistently $85 \pm 5 \%$.

\section{Detection of alpha and SF activities}

Alpha-particle and SF fragment pulse height analyses were performed on each sample for $240 \mathrm{~s}$ using a system of ten $300 \mathrm{~mm}^{2} \mathrm{Si}(\mathrm{Au})$ surface barrier detectors. The pulse heights were digitized and stored in list mode on magnetic tape. Each event was stored along with the time after start of counting and the identification of the detector in which the event occurred. This list-mode data storage allowed versatile off-line processing of the data, including searches for timecorrelated parent-daughter pairs of alpha events in the same detector.

The detector efficiency for the detection of alpha particles was $30 \%$, implying $60 \%$ efficiency for the detection of a fragment from SF decay. Since the ${ }^{262} \mathrm{Ha}$ decay is followed by the alpha decay of its daughter ${ }^{258} \mathrm{Lr}$, the efficiency for detecting the parent $(30 \%)$ and missing the daughter $(70 \%)$ is 0.30 $\times 0.70=0.21$; and likewise for missing the parent and detecting the daughter $0.70 \times 0.30=0.21$. The efficiency for detecting both alpha particles is $0.30 \times 0.30=0.09$. The overall alpha particle detection efficiency is $2 \times 0.21+2 \times 0.09=0.60$. The energy resolution of the surface barrier detectors was $35 \mathrm{keV} \mathrm{FWHM}$ for alpha energies between 5 and $10 \mathrm{MeV}$ in the beginning of the experiments, and deteriorated to about twice that value toward the end.

\section{Results}

Nuclear decay properties of ${ }^{262} \mathrm{Ha}$

In 1597 collection and separation cycles we detected 106 alpha events in the energy range $8.3 \mathrm{MeV}-$ $8.7 \mathrm{MeV}$, compatible with the complex spectra [16] of ${ }^{262} \mathrm{Ha}$ and its daughter, ${ }^{258} \mathrm{Lr}$. 96 fissions attributable, directly or indirectly, to the decay of ${ }^{262} \mathrm{Ha}$ were also detected.

The summed spectrum of the alpha particles observed in those chemical fractions that exhibited ${ }^{262} \mathrm{Ha}$ decays, is shown in Fig. 2. With the counting time of the samples from each separation being four minutes, the spectrum is the result of 106 hours of counting. As a result of incomplete washing of the column after the feeding of the activities onto the column, which was dictated by the need for fast separations, there is a contamination by activities produced (in amounts higher by many orders of magnitude) by transfer reactions of ${ }^{18} \mathrm{O}$ with the ${ }^{249} \mathrm{Bk}$ target (Fm, Md), and with a lead impurity in the target $(\mathrm{Bi}, \mathrm{Po})$. The lower energy part of the spectrum is dominated by peaks at $7.44 \mathrm{MeV}\left({ }^{250} \mathrm{Fm}\right.$ and a little $\left.{ }^{255} \mathrm{Md}\right), 7.19 \mathrm{MeV}$ $\left({ }^{254} \mathrm{Fm}\right.$ and some $\left.{ }^{256} \mathrm{Md}\right), 7.04 \mathrm{MeV}\left({ }^{252} \mathrm{Fm}\right.$ and some $\left.{ }^{255} \mathrm{Fm}\right), \quad 6.62$ and $6.28 \mathrm{MeV}\left({ }^{211} \mathrm{Bi}\right), 6.05 \mathrm{MeV}$ $\left({ }^{212} \mathrm{Bi}\right)$, and $5.81 \mathrm{MeV}\left({ }^{249} \mathrm{Cf}\right)$. The 6.62 and $6.28 \mathrm{MeV}$ peak intensities are in the right ratio for ${ }^{211} \mathrm{Bi}$, and they decay with the ${ }^{211} \mathrm{Bi}$ half-life. The intensity of the $6.05 \mathrm{MeV}$ peak matches well with that of the $8.78 \mathrm{MeV}$ peak. These are ${ }^{212} \mathrm{Bi}$ and ${ }^{212} \mathrm{Po}$, 


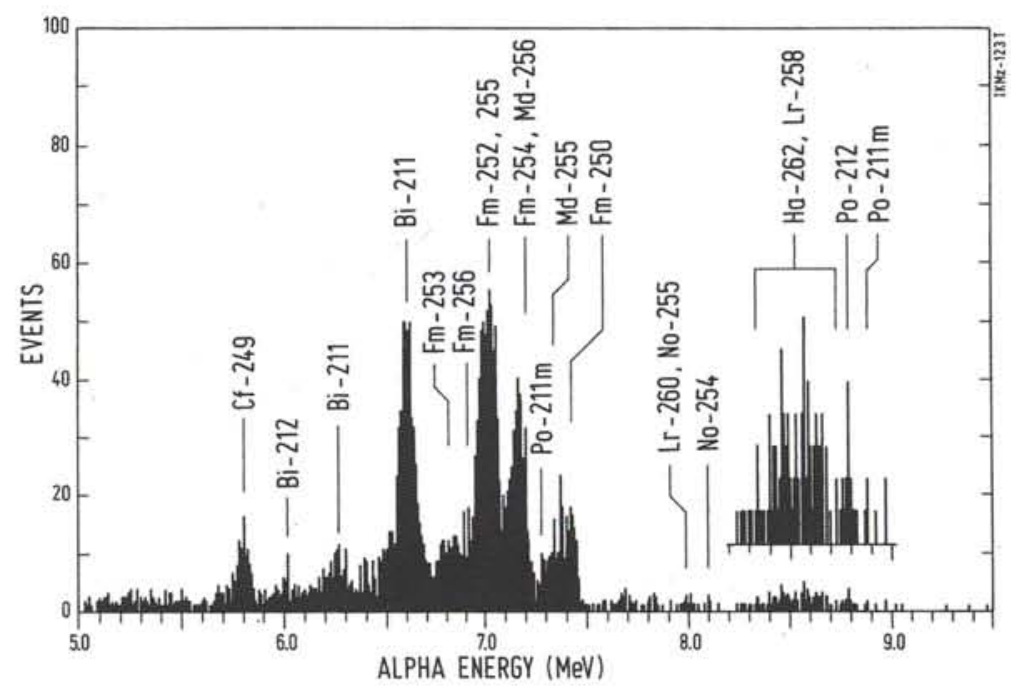

Fig. 2. Sum spectrum of all alpha-particle spectra containing alpha events with energies between 8.3 and $8.7 \mathrm{MeV}$. The vertical scale on the insert of the high energy portion has been expanded.

respectively. The ${ }^{249} \mathrm{Cf}$ is either from the target, or on the detectors from previous experiments.

An important question is, whether the 8.3$8.7 \mathrm{MeV}$ region might be contaminated by $45-\mathrm{s}$ ${ }^{212 \mathrm{~m} 2} \mathrm{Po}(8.52 \mathrm{MeV}$, relative abundance $2 \%)$. There is also a $9.08 \mathrm{MeV}$ peak ( $1 \%$ abundance) of which one can see at most one or two counts in the spectrum. Measuring the $11.65 \mathrm{MeV}$ main activity $(97 \%)$ of ${ }^{212 \mathrm{~m} 2} \mathrm{Po}$ is not possible directly, because it is off the top end of the alpha spectra $(5.0-10.0 \mathrm{MeV})$. However, these appear as overflows in the alpha spectra and at the low end of the fission spectra. In all fractions there were only a few events in this energy range. Therefore, we conclude that less than one count of the $8.52 \mathrm{MeV}$ peak is due to ${ }^{212 \mathrm{~m} 2} \mathrm{Po}$. The lack of intense 7.27 and $8.88 \mathrm{MeV}$ peaks from 25 -s ${ }^{211 \mathrm{~m}}$ Po shows that the decontamination from polonium in the TIOA-column separations was particularly good.

If the spectral range of $8.3-8.7 \mathrm{MeV}$ is clean, there should be the right number of correlated pairs of parent-daughter decays contained in the observed 106 alpha events. From a detector geometry of $30 \%$ of $4 \pi$, one would expect the ratio of correlated pairs to uncorrelated singles to be 0.214 , i.e. one expects to see 15.9 correlated pairs. Fig. 3 illustrates the decay chain [16], and gives a listing of the 15 observed correlations with their energies and times.

We have performed further analyses of the various alpha groups to be assigned to ${ }^{262} \mathrm{Ha}$, and ${ }^{258} \mathrm{Lr}$, respectively, according to Ref. [16], and have looked at their time distributions separately by applying the maximum likelihood method. The results (together with the SF results) are listed in Table 2 . The asymmetric error limits represent the intervals of equal likelihood chances [21] corresponding to a confidence level of $68 \%$.

The singles events of ${ }^{258} \mathrm{Lr}$ appear with the halflife of ${ }^{262} \mathrm{Ha}$. This is because the time from the separation of $\mathrm{Lr}$ from $\mathrm{Ha}$ to the time of the start of counting

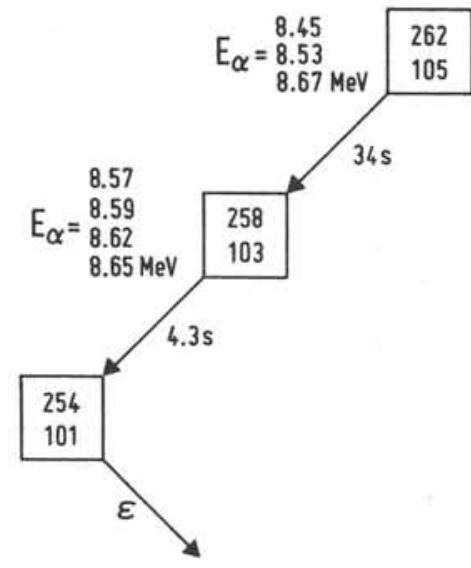

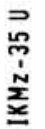

$\alpha-\alpha$ Parent-Daughter Correlations

\begin{tabular}{|r|rr|rr|}
\hline \multirow{2}{*}{ Nr. } & \multicolumn{2}{|c|}{ Parent } & \multicolumn{2}{c|}{ Daughter } \\
& $E_{\alpha} / \mathrm{MeV}$ & $\mathrm{t} / \mathrm{s}$ & $\mathrm{E}_{\alpha} / \mathrm{MeV}$ & \multicolumn{1}{c|}{$\mathrm{t} / \mathrm{s}$} \\
\hline 1 & 8.361 & 20.63 & 8.531 & 15.79 \\
2 & 8.665 & 25.80 & 8.571 & 11.10 \\
3 & 8.460 & 39.68 & 8.659 & 0.24 \\
4 & 8.509 & 149.73 & 8.562 & 7.24 \\
5 & 8.344 & 2.77 & 8.577 & 11.60 \\
6 & 8.513 & 88.60 & 8.641 & 2.45 \\
7 & 8.397 & 157.22 & 8.664 & 3.61 \\
8 & 8.455 & 1.50 & 8.593 & 7.07 \\
9 & 8.689 & 21.53 & 8.656 & 19.69 \\
10 & 8.479 & 10.67 & 8.596 & 6.81 \\
11 & 8.465 & 23.62 & 8.592 & 13.26 \\
12 & 8.357 & 23.90 & 8.521 & 2.65 \\
13 & 8.406 & 49.55 & 8.570 & 0.03 \\
14 & 8.605 & 146.87 & 8.655 & 9.21 \\
15 & 8.476 & 1.77 & 8.461 & 21.18 \\
\hline
\end{tabular}

Fig. 3. The decay chain of ${ }^{262} \mathrm{Ha}[16]$ and a list of the alphaalpha parent-daughter correlations observed in the hahnium experiments. The times of the parent events are the times from the beginning of counting to the alpha event. The times of the daughter events are the times that elapsed since the parent event. 
was long enough so that the ${ }^{258} \mathrm{Lr}$ was (almost) in secular equilibrium with the ${ }^{262} \mathrm{Ha}$ parent. The halflives determined for ${ }^{262} \mathrm{Ha}$ singles, ${ }^{258} \mathrm{Lr}$ singles, ${ }^{262} \mathrm{Ha}$ correlated mothers, and the fissions are all consistent with the known half-life of $34 \pm 4 \mathrm{~s}$ for ${ }^{262} \mathrm{Ha}$ [16]. The half-life of the ${ }^{258} \mathrm{Lr}$ correlated daughters is slightly longer than the known half-life of $4.3 \pm 0.5 \mathrm{~s}$ [16]. This discrepancy may be due to the effect of random correlation of unrelated events and will be discussed further below.

Table 2 shows that we may have observed an unknown alpha emitter at $8.49 \mathrm{MeV}$. The average life time of the 15 events is about $120 \mathrm{~s}$, indicating a random distribution during the counting interval of $240 \mathrm{~s}$. Therefore, the half-life of this component may be much longer than the numerical value listed in Table 2. The long life time seems to suggest that there is an extra activity not due to the decay of $34-\mathrm{s}^{262} \mathrm{Ha}$. There are several possible origins of the $8.49 \mathrm{MeV}$ activity:

i) ${ }^{262} \mathrm{Ha}$ and $/$ or ${ }^{258} \mathrm{Lr}$ could have isomers.

ii) ${ }^{263} \mathrm{Ha}$ and/or its daughter ${ }^{259} \mathrm{Lr}$ could be showing up here.

${ }^{263} \mathrm{Ha}$ has never been observed in spite of the fact that evaporation calculations predict a cross section for the ${ }^{249} \mathrm{Bk}\left({ }^{18} \mathrm{O}, 4 \mathrm{n}\right)$ reaction producing ${ }^{263} \mathrm{Ha}$ very similar to the cross section for the ${ }^{249} \mathrm{Bk}\left({ }^{18} \mathrm{O}, 5 \mathrm{n}\right)$ ${ }^{262} \mathrm{Ha}$ reaction near $100 \mathrm{MeV}$ bombarding energy.

iii) It is possible to discuss another isomer in the $\mathrm{Bi}$ or Po isotopes.

This seems to be the least likely because in order to have this interference from an unknown isomer of a $\mathrm{Bi}$ or Po isotope, one should have seen more from the known isomers. Other experiments are necessary to clarify the origin of the $8.49 \mathrm{MeV}$ activity.

If 15 events out of 106 events in the energy range that covers the ${ }^{262} \mathrm{Ha}$ and ${ }^{258} \mathrm{Lr}$ spectra are due to something else, we have detected $91{ }^{262} \mathrm{Ha} /{ }^{258} \mathrm{Lr}$ events. Assuming the ${ }^{262} \mathrm{Ha}$ and ${ }^{258} \mathrm{Lr}$ decays reached secular equilibrium at the time of the start of counting and thereafter the activity of the daughter, $A_{\mathrm{d}}$, was related to the parent activity, $A_{\mathrm{p}}$, by the following relation:

$$
A_{\mathrm{d}}=\frac{\lambda_{\mathrm{d}}}{\lambda_{\mathrm{d}}-\lambda_{\mathrm{p}}} A_{\mathrm{p}}
$$

where $\lambda_{\mathrm{d}}$ and $\lambda_{\mathrm{p}}$ are the decay constants of daughter and parent, respectively.

Inserting the known decay constants gives $A_{\mathrm{d}}=$ $1.14 A_{\mathbf{p}}$. This means, ignoring statistical fluctuations, that 48.5 events are due to ${ }^{258} \mathrm{Lr}$, and 42.5 events are due to ${ }^{262} \mathrm{Ha}$. At a detector geometry of $30 \%$ of $4 \pi$, we expect to see $42.5 \times 0.3=12.8$ true parent daughter correlations. With 106 total events and 12.8 parentdaughter correlations, we observed the decay of $106-12.8=93.2$ atoms. These atoms were distributed randomly among 1597 experiments. In 536 of these experiments, the ${ }^{262} \mathrm{Ha}$ events were evenly distributed between two chemical fractions which were counted simultaneously. This makes the number of
Table 2. Half-lives of the various components in the alpha spectra in the energy range 8.3 through $8.7 \mathrm{MeV}$ and of the SF activity after subtraction of a background due to ${ }^{256} \mathrm{Md} /{ }^{256} \mathrm{Fm}$

\begin{tabular}{lcc}
\hline Species & Half-life (s) & Events \\
\hline${ }^{262} \mathrm{Ha}$ singles & $39.8 \pm 8.9$ & 30 \\
${ }^{258} \mathrm{Lr}$ singles & $36.0 \pm 7.9$ & 31 \\
${ }^{262} \mathrm{Ha}$ corr. mothers & $35.3 \pm 12.3$ & 15 \\
& $6.1 \pm 2.1$ & 15 \\
${ }^{258} \mathrm{Lr}$ corr. daughters & $33.5 \pm 9.4$ & 96 \\
Fissions & $83.9 \pm 29.2$ & 15 \\
$8.49 \mathrm{MeV} \alpha$-activity & 17.2 & \\
\hline
\end{tabular}

counting intervals among which the events were randomly distributed 2133 . The expected number of random correlations of unrelated alpha particles, assuming Poisson statistics, is 1.95 . The predicted 12.8 true correlations and random correlations give a total of about 15 expected correlated pairs in agreement with the observed number of "correlated" events. (If the 15 events at $8.49 \mathrm{MeV}$ are included as being due to $34-\mathrm{s}{ }^{262} \mathrm{Ha} /{ }^{258} \mathrm{Lr}$, the number of expected parent daughter correlations is 15.9 and the expected number of random correlations is 1.82 , giving a total of 17.7 , which is also consistent with the 15 observed correlations.)

The alpha-alpha correlations with daughter energies below $8.52 \mathrm{MeV}$ may be due to one of two things: a) Random correlations. Event No. 15 with $E_{\mathrm{p}}=$ $8.476 \mathrm{MeV}$ and $E_{\mathrm{d}}=8.461 \mathrm{MeV}$ is probably a pair of two ${ }^{262} \mathrm{Ha}$ decays in one sample. This is further supported by the fact that the apparent daughter life time was $21.18 \mathrm{~s}$. This is very long for ${ }^{258} \mathrm{Lr}$, but $21.18 \mathrm{~s}+1.77 \mathrm{~s}=22.95 \mathrm{~s}$ is very reasonable for ${ }^{262} \mathrm{Ha}$. If we exclude this event from the maximum likelihood analysis of the ${ }^{258} \mathrm{Lr}$ life times we obtain, instead of a half life of $6.1+2.1-1.3 \mathrm{~s}$, a value of $5.5+2.0-1.2 \mathrm{~s}$ which is in agreement within error limits with the literature half-life [16] of $4.3 \pm 0.5 \mathrm{~s}$. (b) The low energy events could have occurred in some experiments where the counting samples were not ideally weightless. A number of "low" parent energies $(8.3-8.4 \mathrm{MeV})$ is likely to be caused by the same reason.

We also observed a total of 109 SF decays. Some of these are due to contamination by ${ }^{256} \mathrm{Md} /{ }^{256} \mathrm{Fm}$. This fraction can be estimated by independent methods: i) a decay-curve analysis, see Fig. 4 , ii) from the known alpha-to-SF ratio of ${ }^{256} \mathrm{Md} /{ }^{256} \mathrm{Fm}$ and the $\mathrm{Fm} / \mathrm{Md}$ contamination visible in Fig. 2 and iii) from the ratio of SF to ${ }^{252,255} \mathrm{Fm}$ activity in the gold catcher foil experiments mentioned in Section 3, and the ${ }^{252,255} \mathrm{Fm}$ visible in Fig. 2. All methods consistently give 13 events for the contribution of ${ }^{256} \mathrm{Md} /{ }^{256} \mathrm{Fm}$. The solid line in Fig. 4 is a least squares fit with fixed 


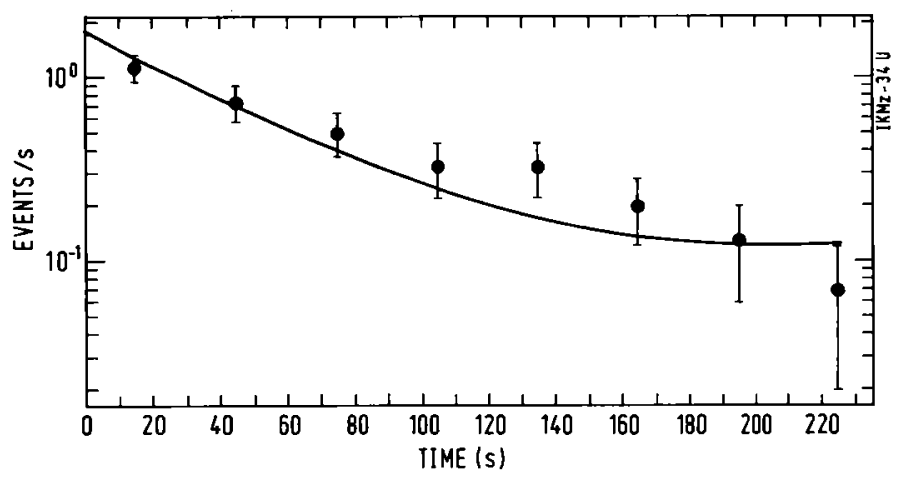

Fig. 4. Decay curve of the SF activity of chemically isolated hahnium. The solid line results from a least squares fit with a long-lived component $\left({ }^{256} \mathrm{Md} /{ }^{256} \mathrm{Fm}\right)$ and a short component of $33.5 \mathrm{~s}$ half-life.

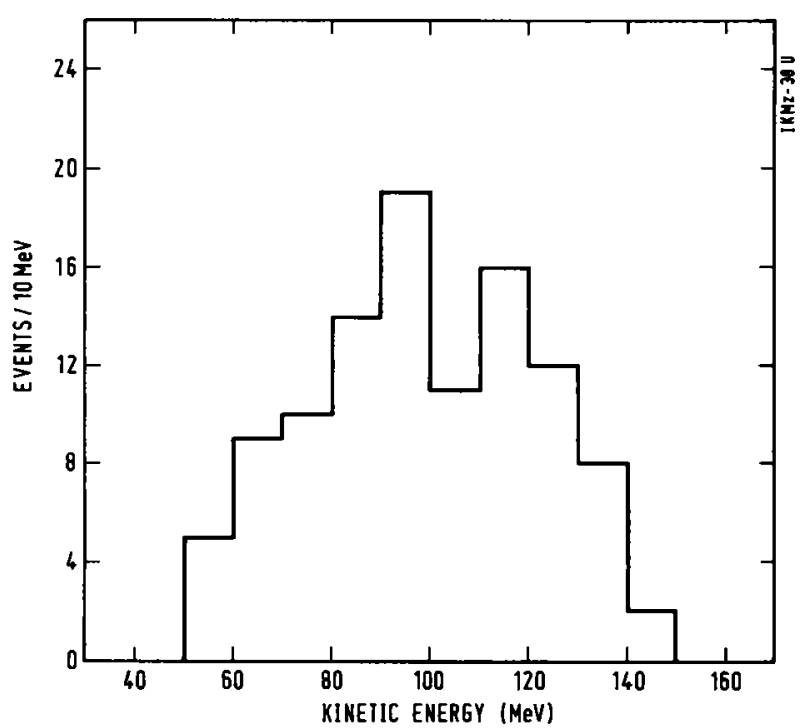

Fig. 5. Fission fragment kinetic energy spectrum.

half-lives for the long-lived components $(1.3 \mathrm{~h}, 2.63 \mathrm{~h})$ and gives a half-life of $33.5 \pm 9.4 \mathrm{~s}$ for the component associated with the decay of ${ }^{262} \mathrm{Ha}$.

Assuming that SF is a decay mode of $34-\mathrm{s}{ }^{262} \mathrm{Ha}$, we can calculate the branching ratio. The $8.49 \mathrm{MeV}$ alphas are excluded as not due to $34-\mathrm{s}^{262} \mathrm{Ha}$. The SF branch is then $51 \pm 11 \%$ in agreement with Ref. [3].

Fig. 5 shows the fission fragment kinetic energy spectrum. It is important to note that only single SF fragments were detected, so that conclusions about the mass distribution and the total kinetic energy are difficult. The energy calibration is approximate and was made by comparison to the fission spectrum from $a^{252} \mathrm{Cf}$ source measured in the same detectors. Under the assumption that there is only one mode of fission, the average total kinetic energy appears to be close to $200 \mathrm{MeV}$. Also, it appears that the compact scission mode leading to high total kinetic energies of about $233 \mathrm{MeV}$ as observed in some $\mathrm{Fm}$ and $\mathrm{Md}$ isotopes [22] is not dominant. Fits of the distribution with one Gaussian $(\langle E\rangle=99.5 \mathrm{MeV}, \mathrm{FWHM}=53.2 \mathrm{MeV})$, or with two Gaussians of equal height and width $\left(\left\langle E_{1}\right\rangle=82.9 \mathrm{MeV},\left\langle E_{2}\right\rangle=117.0 \mathrm{MeV}\right.$, FWHM $=$
$40.3 \mathrm{MeV}$ ), are of similar quality. Thus we cannot decide from the present data whether the distribution is asymmetric, as suggested by Bemis et al. [12], or symmetric.

From the ${ }^{249} \mathrm{Bk}$ content in the target, the $\mathrm{He} /$ $\mathrm{KCl}$-jet efficiencies, the counting efficiencies, the beam currents, and the number of ${ }^{262} \mathrm{Ha}$ decays detected, and with the assumption that the chemical yield for hahnium was the same as for niobium, tantalum, and protactinium, we obtain a cross section of $4.1 \pm 1.2 \mathrm{nb}$ for the alpha activity of ${ }^{262} \mathrm{Ha}$, giving a total cross section of $8.3 \pm 2.4 \mathrm{nb}$ at $98-99 \mathrm{MeV}$ bombarding energy. This is more than a factor of two greater than reported in Ref. [3] for $101 \mathrm{MeV}$. The discrepancy is probably due to an overestimate of the gas jet and/or chemical yield in Ref. [3] or a systematic underestimate of the gas jet yield in this work.

To conclude, we have detected a statistically significant number of alpha and SF decays that can be uniquely assigned to $34-\mathrm{s}{ }^{262} \mathrm{Ha}$. The alpha particle energies (within resolution), the half-lives, the number of observed parent-daughter correlations relative to the number of singles, as well as the SF decay branch of $51 \%$, are highly consistent with each other, and with the most recent results [3]. On the basis of this unique identification, it is possible to draw conclusions about the chemical properties of element 105 in the present experiments, even if only a few atoms were detected in a given chemical fraction.

\section{Extraction chromatography with TIOA}

In the beginning of the TIOA extraction experiments, it was most important to verify that hahnium halide complexes were extracted into the amine phase under the same conditions as niobium, tantalum, and protactinium were extracted. According to the results presented in Section 2 this could be achieved either from $12 \mathrm{M} \mathrm{HCl} / 0.02 \mathrm{M} \mathrm{HF}$, or from $10 \mathrm{M} \mathrm{HCl}$ solutions. The extraction was verified by feeding the activities to the columns, and by stripping the amine (along with the extracted activities) from the columns by dissolving it in acetone. The acetone strip fraction was evaporated to dryness, and assay of the samples for alpha- 


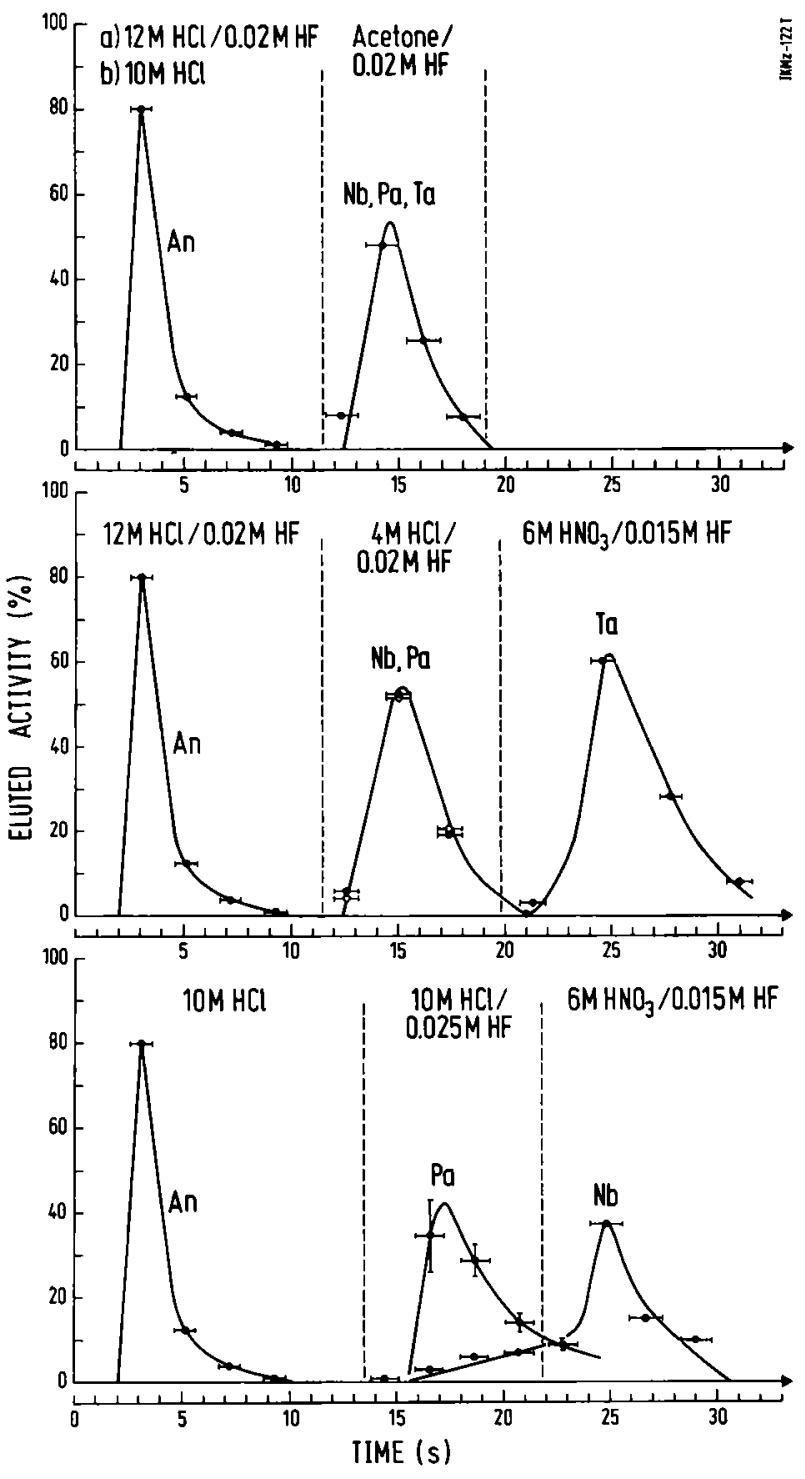

Fig. 6. Elution curves for carrier-free actinides (An), Nb, Ta, and $\mathrm{Pa}$ from TIOA/Voltalef ${ }^{\mathrm{R}}$ columns $(1.6 \times 8 \mathrm{~mm})$ under the same conditions as in the hahnium experiments. The horizontal error bars are associated with uncertainties in converting drop numbers into effluent volumes, i.e. times. In the upper part the $\mathrm{Ha}$ activity was adsorbed onto the column from a) $12 \mathrm{M} \mathrm{HCl} /$ $0.02 \mathrm{M} \mathrm{HF}$, b) $10 \mathrm{M} \mathrm{HCl}$, and stripped along with the TIOA from the column in acetone/0.02 M HF. In the middle part the activity was extracted as in a), followed by separate elutions of a Nb-Pa fraction, and a Ta fraction. In the lower part the activity was extracted as in b), followed by a $\mathrm{Pa}$, and then a $\mathrm{Nb}$ fraction.

particle and SF decay started about $45 \mathrm{~s}$ after the end of the collection cycle. Elution curves for actinides, $\mathrm{Nb}, \mathrm{Ta}$, and $\mathrm{Pa}$ under the same conditions, with carrier-free activities produced on-line, as described in Section 3, are shown in the top section of Fig. 6. In a first series of 207 experiments, extraction into the column material occurred from $12 \mathrm{M} \mathrm{HCl} / 0.02 \mathrm{M}$ $\mathrm{HF}$, and $9{ }^{262} \mathrm{Ha} /{ }^{258} \mathrm{Lr}$ alpha events, including one correlated pair, and $12 \mathrm{SF}$ decays were recorded, indicating that ${ }^{262} \mathrm{Ha}$ had formed anionic complexes that were extracted by TIOA.

In a second series of 133 experiments, extraction into the amine phase occurred from $10 \mathrm{M} \mathrm{HCl}$. This time, $2{ }^{262} \mathrm{Ha} /{ }^{258} \mathrm{Lr}$ alpha events and $4 \mathrm{SF}$ decays were detected in the acetone strip fraction. This demonstrated that hahnium behaved similar to $\mathrm{Nb}, \mathrm{Ta}$, and $\mathrm{Pa}$, also in $10 \mathrm{M} \mathrm{HCl}$. The yield of ${ }^{262} \mathrm{Ha}$ events in this latter series seems to be somewhat low. However, this was not caused by incomplete extraction of $\mathrm{Ha}$ into the amine, as was shown later, but presumably by a low $\mathrm{He} / \mathrm{KCl}$-jet efficiency. The latter had not been checked in this series.

In the next series of 721 collection and separation cycles, the activity was adsorbed from $12 \mathrm{M} \mathrm{HCl} /$ $0.02 \mathrm{M} \mathrm{HF}$ on the TIOA column as before. A Nb-Pa fraction was removed from the column with $4 \mathrm{M} \mathrm{HCl}$ / $0.02 \mathrm{M} \mathrm{HF}$, followed by the stripping of Ta from the column in $6 \mathrm{M} \mathrm{HNO}_{3} / 0.015 \mathrm{M} \mathrm{HF}$, as shown in the middle part of Fig. 6. On the average, there was a tailing of about $10 \%$ of the $\mathrm{Nb} / \mathrm{Pa}$ activity into the $\mathrm{Ta}$ fraction. $88 \%$ of the ${ }^{262} \mathrm{Ha} /{ }^{258} \mathrm{Lr}$ alpha activity (38 events, including 4 alpha-alpha correlations) were found in the $\mathrm{Nb}-\mathrm{Pa}$ fraction, and $12 \%$ (5 events, with no correlated pair) in the Ta fraction. This distribution is identical with the behavior of $\mathrm{Nb}$ and $\mathrm{Pa}$, and distinctly different from that of Ta. This shows, that the anionic halide complexes of $\mathrm{Ha}$ are not as strong as for $\mathrm{Ta}$, and are more like those of $\mathrm{Nb}$ or $\mathrm{Pa}$. Apparently, in contrast to simple extrapolations, the trend in the chemical properties of the group-VB elements from $\mathrm{Nb}$ to Ta does not continue but is reversed in going from $\mathrm{Ta}$ to $\mathrm{Ha}$. The distribution of SF decays between the Nb-Pa fraction (39) and the Ta fraction (10) corroborates the above finding.

In a last series of 536 experiments, a separation of $\mathrm{Pa}$ from $\mathrm{Nb}$ was performed (see bottom part of Fig. 6). After feeding of the activities in $10 \mathrm{M} \mathrm{HCl}$ onto the column, Pa was eluted first with $10 \mathrm{M} \mathrm{HCl}$ / $0.025 \mathrm{M}$ HF. Under these conditions a fraction of the $\mathrm{Nb}$ activity begins to break through. The change of the eluent to $6 \mathrm{M} \mathrm{HNO}_{3} / 0.015 \mathrm{M} \mathrm{HF}$ was timed such that the $\mathrm{Pa}$ fraction contained $80 \%$ of the $\mathrm{Pa}$ and $20 \%$ of the $\mathrm{Nb}$, while the $\mathrm{Nb}$ fraction contained the remaining $20 \%$ of $\mathrm{Pa}$ and $80 \%$ of the $\mathrm{Nb}$ activity. The ${ }^{262} \mathrm{Ha}$ decays were equally divided between the $\mathrm{Pa}$ and $\mathrm{Nb}$ fractions: There were 25 alpha events, including 5 correlated pairs, in the $\mathrm{Pa}$ fraction, and 27 alpha events, including 5 correlated pairs, in the $\mathrm{Nb}$ fraction. $\mathrm{The} \mathrm{Pa} / \mathrm{Nb}$ ratio of SF decays was $25: 19$. The results indicate that the halide complexing strength in $\mathrm{Ha}$ is between that for $\mathrm{Pa}$ and $\mathrm{Nb}$.

A summary of the distribution of alpha and SF decays among the various chemical fractions is contained in Table 3. The reason for listing SF decays separately for times larger than $140 \mathrm{~s}$ is as follows: After this time, the ${ }^{262} \mathrm{Ha}$ activity has decreased to about $5 \%$ of the initial activity, and the contribution by ${ }^{256} \mathrm{Md} /{ }^{256} \mathrm{Fm}$ becomes dominant. Thus, the events listed in the last column of Table 3 might give some idea about the distribution of SF-background events. The distribution of the unknown $8.49 \mathrm{MeV}$ alpha activity among the fractions is similar to that of the ${ }^{262} \mathrm{Ha}$ events. 
Table 3. Observed 8.3 - 8.7 MeV alpha decays and SF decays in the chemical fractions containing Nb, Ta, and/or Pa. The unknown $8.49 \mathrm{MeV}$ alpha events and SF events with life-times $>140 \mathrm{~s}$ are shown separately

\begin{tabular}{|c|c|c|c|c|c|c|}
\hline $\begin{array}{l}\text { Feeding } \\
\text { solution }\end{array}$ & $\begin{array}{l}\text { Chemical } \\
\text { fraction }\end{array}$ & $\begin{array}{c}\text { Total } \\
\text { alpha } \\
\text { events }\end{array}$ & $\begin{array}{c}\text { Correlated } \\
\text { pairs }\end{array}$ & $\begin{array}{c}8.49 \mathrm{MeV} \\
\text { alpha } \\
\text { events }\end{array}$ & $\begin{array}{c}\text { Total } \\
\text { SF decays }\end{array}$ & $\begin{array}{c}\mathrm{SF}^{\mathrm{b}} \\
\text { decays }\end{array}$ \\
\hline $\begin{array}{l}12 \mathrm{M} \mathrm{HCl} / \mathrm{HF} \\
10 \mathrm{M} \mathrm{HCl}\end{array}$ & $\begin{array}{l}\text { acetone } \\
\text { acetone }\end{array}$ & $\begin{array}{l}9 \\
2\end{array}$ & $\begin{array}{l}1 \\
0\end{array}$ & $\begin{array}{l}2 \\
1\end{array}$ & $\begin{array}{r}12 \\
4\end{array}$ & $\begin{array}{l}0 \\
1\end{array}$ \\
\hline $12 \mathrm{M} \mathrm{HCl} / \mathrm{HF}$ & $\begin{array}{l}\mathrm{Nb}-\mathrm{Pa} \\
\mathrm{Ta}\end{array}$ & $\begin{array}{r}38 \\
5\end{array}$ & $\begin{array}{l}4 \\
0\end{array}$ & $\begin{array}{l}7 \\
0\end{array}$ & $\begin{array}{l}39 \\
10\end{array}$ & $\begin{array}{l}6 \\
0\end{array}$ \\
\hline $10 \mathrm{M} \mathrm{HCl}$ & $\begin{array}{l}\mathrm{Pa} \\
\mathrm{Nb}\end{array}$ & $\begin{array}{l}25 \\
27\end{array}$ & $\begin{array}{l}5 \\
5^{\mathrm{a}}\end{array}$ & $\begin{array}{l}3 \\
1\end{array}$ & $\begin{array}{l}25 \\
19\end{array}$ & $\begin{array}{l}3 \\
2\end{array}$ \\
\hline
\end{tabular}

Among those is one random correlation between two ${ }^{262} \mathrm{Ha}$ decays

b Life time $>140 \mathrm{~s}$

\section{Conclusions and discussion}

The 34-s ${ }^{262} \mathrm{Ha}$ was observed in chemical experiments in which hahnium was extracted from strong $\mathrm{HCl}$ or $\mathrm{HCl} / \mathrm{HF}$ solutions into triisooctyl amine. The identification of the ${ }^{262} \mathrm{Ha}$ was based on the observation of alpha particles due to the decay of ${ }^{262} \mathrm{Ha}$ and its daughter, ${ }^{258} \mathrm{Lr}$. Both the alpha-particle energies and the times at which the events occurred were used as identification criteria. Also, time-correlated pairs of alpha particles from the decay of ${ }^{262} \mathrm{Ha}$ and its daughter were recorded. There is some evidence for a longer-lived $8.49 \mathrm{MeV}$ alpha activity from the decay of another isotope (or isomer) of hahnium, and/or from a daughter of a new hahnium isotope or isomer.

The alpha branch for $34-\mathrm{s}{ }^{262} \mathrm{Ha}$ has been found to be $49 \pm 10 \%$ in agreement with Ref. [3], with the remainder of the decay being either by SF or by electron capture to $60-\mathrm{ms}{ }^{262} \mathrm{Rf}$ which then decays by $\mathrm{SF}$. The fission fragment kinetic energy spectrum is a broad distribution with a total kinetic energy close to $200 \mathrm{MeV}$.

The total cross section for the formation of 34-s ${ }^{262} \mathrm{Ha}$ in the ${ }^{249} \mathrm{Bk}\left({ }^{18} \mathrm{O}, 5 \mathrm{n}\right)$ reaction at the laboratory energy of $98-99 \mathrm{MeV}$ is $8.3 \pm 2.4 \mathrm{nb}$.

Hahnium is extracted into TIOA from $12 \mathrm{M} \mathrm{HCl} /$ $0.02 \mathrm{M} \mathrm{HF}$ as well as from $10 \mathrm{M} \mathrm{HCl}$ solutions, like $\mathrm{Nb}, \mathrm{Ta}$, and $\mathrm{Pa}$. This indicates that the most stable oxidation state for hahnium is also +5 , and that $\mathrm{Ha}^{5+}$ forms anionic halide complexes, a chemical property characteristic of the group-VB elements. In elutions with $4 \mathrm{M} \mathrm{HCl} / 0.02 \mathrm{M} \mathrm{HF}$ (Nb-Pa fraction) and with $6 \mathrm{M} \mathrm{HNO}_{3} / 0.015 \mathrm{M} \mathrm{HF}$ (Ta fraction), the ${ }^{262} \mathrm{Ha}$ activity was found in the $\mathrm{Nb}-\mathrm{Pa}$ fraction showing that the anionic halide complexes of hahnium are not as strong as are those of tantalum, and are more like those of niobium and protactinium. In elutions with $10 \mathrm{M} \mathrm{HCl} / 0.025 \mathrm{M} \mathrm{HF}$ (Pa fraction) and $6 \mathrm{M} \mathrm{HNO}_{3} /$ $0.015 \mathrm{M} \mathrm{HF}$ (stripping of $\mathrm{Nb}$ ) the ${ }^{262} \mathrm{Ha}$ was found to be equally divided between the protactinium and niobium fractions, i.e., the distribution constant of hahnium in the $10 \mathrm{M} \mathrm{HCl} / 0.025 \mathrm{M} \mathrm{HF}$ - TIOA system is between those of protactinium and niobium.
The non-tantalum like behaviour of hahnium halide complexes observed earlier [3] can be understood in the frame of the results of this work: Because the halide complexes of hahnium are like those of niobium, and not like those of tantalum, hahnium was not extracted by MIBK [3] as the procedure was specific for tantalum and did not include a niobiumlike behaviour.

In strong hydrofluoric acid solutions, niobium is reported to be present as the oxofluoro complex $\left[\mathrm{NbOF}_{5}\right]^{2-}$, while tantalum forms $\left[\mathrm{TaF}_{6}\right]^{-}$and $\left[\mathrm{TaF}_{7}\right]^{2-}$ complexes [23]. These species were identified by Raman and ${ }^{19} \mathrm{~F}$-NMR spectroscopy. The formation of $\left[\mathrm{NbOCl}_{4}\right]^{-}$in weaker $\mathrm{HCl}$ and $\left[\mathrm{NbOCl}_{5}\right]^{2-}$ in strong $\mathrm{HCl}$ solutions as a basis for their amine separations from the tantalum complexes $\left[\mathrm{TaCl}_{6}\right]^{-}$ and $\left[\mathrm{TaCl}_{7}\right]^{2-}$ is well known $[24,25]$. The predominance of oxochloro (or hydroxochloro) complexes in the protactinium chemistry, similar to niobium, is also known [26]: In $3 \mathrm{M} \mathrm{HCl}$ protactinium is present as $\left[\mathrm{PaOCl}_{4}\right]^{-}$(or $\left[\mathrm{Pa}(\mathrm{OH})_{2} \mathrm{Cl}_{4}\right]^{-}$) and not as $\left[\mathrm{PaCl}_{6}\right]^{-}$; in $>6 \mathrm{M} \mathrm{HCl}$, complexes such as $\left[\mathrm{PaOCl}_{5}\right]^{2-}$, $\left[\mathrm{Pa}(\mathrm{OH})_{2} \mathrm{Cl}_{5}\right]^{2-}$, or $\left[\mathrm{Pa}(\mathrm{OH}) \mathrm{Cl}_{6}\right]^{-}$are still predomi1lithl. I he largely parallel extraction behaviour of niobium and protactinium in strong $\mathrm{HCl}$ and $\mathrm{HCl}$ / $0.03 \mathrm{M} \mathrm{HF}$ solutions (Fig. 1e), very different from the extraction behaviour of tantalum, is likely to be connected with the structural difference of their halide complexes. It is then tempting to suggest, on the basis of the very similar extraction behaviour of hahnium with that of niobium and protactinium, that the structure of the hahnium halide complexes is of the oxohalide (or hydroxohalide) type, e.g., $\left[\mathrm{HaOCl}_{4}\right]^{-}$ and $\left[\mathrm{HaOCl}_{5}\right]^{2-}$, as for niobium and protactinium. This should be investigated further by performing hahnium extractions from $\mathrm{HCl}$ concentrations lower than $4 \mathrm{M}$, i.e., from $0.5 \mathrm{M} \mathrm{HCl}$, see Figs. $1 \mathrm{~b}$ and/or 1e, where the extraction yield should go up again, and from $0.1 \mathrm{M} \mathrm{HCl}$, where the extraction should be poor, if the close analogy to the behaviour of $\mathrm{Nb}$ and $\mathrm{Pa}$ persists also at these acid concentrations.

Such structural differences, which are also known for the group-VIB elements and the higher groups, 
must be connected to the electronic structure of the metal ions involved. For the +5 ions of group VB we have the following electronic configurations: i) $[\mathrm{Kr}]$ for niobium, with the empty $4 d$ orbitals being essential for complex formation; ii) [Xe] $4 f^{14}$ for tantalum with the $5 d$ orbitals being involved; iii) [Rn] for protactinium with the $6 d$ orbitals participating in the ligand bonds; and iv) $[R n] 5 f^{14}$ for hahnium, where again the $6 d$ orbitals are essential in the complex formation. In order to understand the different structures it seems to be essential to look into the properties of the nd wave functions and their specific role in the quantum chemistry of molecules. Trends in other properties of these ions, i.g., their ionic radii, are rather monotonic [27] and would not suggest reversed trends in going from $\mathrm{Nb}$ via $\mathrm{Ta}$ to $\mathrm{Pa}$ and Ha. Pyykkö has recently reviewed relativistic effects in structural chemistry and has emphasized the critical dependence of ligand bonding on the radial extension of $4 d, 5 d$, and $6 d$ wave functions in the respective molecules [28, 29]. These radial extensions depend primarily on the filling of the different electron shells and the different number of nodes in them. In addition, already the difference between the $4 d$ and $5 d$ orbitals, seems to be due to large, if not dominant, relativistic contributions which, however, enter in an individualistic manner for the various groups of elements [29]. For example, Pyykkö discusses the radial electron distribution for the $(4 d)^{4}$ shell and the corresponding $(5 d)^{4}$ shell in the octahedral model systems $\mathrm{MoH}_{6}$ and $\mathrm{WH}_{6}$ and finds that the relativistic $5 d$ distribution for $\mathrm{WH}_{6}$ has a main maximum that has moved from about 2 atomic units (in $\mathrm{MoH}_{6}$ ) to around $3-3.5$ atomic units in $\mathrm{WH}_{6}$ due to relativistic effects, see Fig. 3 in Ref. [29]. He discusses similar effects in $\mathrm{MoO}_{3}$ and $\mathrm{WO}_{3}$ [28], and he points out, that the smaller $\mathrm{Mo}(4 d)-\mathrm{O}(2 p)$ overlap in $\mathrm{MoO}_{3}$ leads to a displacement of the Mo ions in the crystal lattice and to the stabilization of a layered structure. In $\mathrm{WO}_{3}$ the $5 d$ functions are much more diffuse than the $4 d$ in $\mathrm{MoO}_{3}$ leading to a larger W(5d)$\mathrm{O}(2 p)$ overlap, and to a smaller distortion, avoiding the formation of $[\mathrm{W}=\mathrm{O}]^{4+}$ ions. If similar trends hold for the neighbouring group-VB elements one might view the situation as follows: The relatively small radial extension of the $4 d$ wave functions in $\mathrm{Nb}$ favors the formation of $[\mathrm{Nb}=\mathrm{O}]^{3+}$ oxoniobium cations with a relatively short $\mathrm{Nb}-\mathrm{O}$ bond length. Both the compactness of the $4 d$ orbitals as well as the presence of the oxygen bond might then make the $4 d$ orbitals less accessible and diminish their contribution to ligand bonding. Due to the relativistic expansion of the $5 d$ shell in tantalum, there is a larger overlap of orbitals and a stronger bonding even with larger ligands such as $\mathrm{Cl}^{-}$, so that the pure $\left[\mathrm{TaCl}_{6}\right]^{-}$or $\left[\mathrm{TaCl}_{7}\right]^{2-}$ complexes might be more stable than the oxochloride forms. This trend is not necessarily to continue when proceeding to the $6 d$ orbitals in protactinium and hahnium. Pyykkö has pointed out that the relativistic contraction of $s$ and $p$ orbitals and the relativistic expansion of the $d$ orbitals may have two diametrically opposed consequences depending on whether the orbital energy of the $d$ orbital is above or below those of the other valence orbitals [29]. In the former case, possibly relevant for the $6 d$ participation in the bonds of $\mathrm{Pa}$ and $\mathrm{Ha}$, the relativistic effects make the $6 d$ orbitals energetically less accessible. In the latter case, relevant for the $5 d$ participation in ligand bonds in tantalum, the relativistic effects push the $d$ orbitals up in energy and outwards in space, diminishing their isolation in the atomic core and increasing their contribution to the bonds, and strengthening the latter [29]. A very interesting example for such very individualistic relativistic contributions can be found in the extreme chemical similarity of $\mathrm{Zr}$ and $\mathrm{Hf}$ [29]: It is suggested that the chemical similarity of $\mathrm{Zr}$ and $\mathrm{Hf}$ is due to a local, anomalous cancellation of relativistic effects against shell-structure trends.

This discussion, as well as the rather unexpected experimental results of this work, suggest that the chemical properties of the heaviest elements cannot reliably be predicted by simple extrapolations of trends within a group of elements, but that the properties depend locally on subtle, individual differences in the configuration mixing of the bonding orbitals. Relativistic, quantum chemical calculations for compounds of $\mathrm{Nb}, \mathrm{Ta}, \mathrm{Pa}$, and $\mathrm{Ha}$ are needed to understand in detail the differences in the halide complexing of the group-VB elements.

\section{Acknowledgements}

The authors are indebted to the Division of Chemical Sciences, Office of Basic Energy Sciences, U.S. Department of Energy, for making the ${ }^{249} \mathrm{Bk}$ target material available through the transplutonium element production facilities at the Oak Ridge National Laboratory.

We like to thank Dr. N. Trautmann and S. Zauner for advice and help, and the staff and crew of the Mainz TRIGA reactor for assistance in the test phase of the experiment.

We wish to thank the staff and crew of the LBL 88-inch Cyclotron for providing the ${ }^{18} \mathrm{O},{ }^{20} \mathrm{Ne}$ and ${ }^{4} \mathrm{He}$ beams and for technical support.

The German and Swiss groups have enjoyed the hospitality of the Lawrence Berkeley Laboratory $\mathrm{Nu}$ clear Science Division.

This work was supported by the German Federal Minister for Research and Technology (BMFT) under contract No. 03-HE1MAI and 03-HE2MAI, by the Director, Office of Energy Research, Office of Basic Energy Sciences, Chemical Sciences Division of the U.S. Department of Energy under Contract No. DEAC03-76SF00098, and by the Swiss National Science Foundation under Contract No. 2.204-0.86. H.L.H. was supported by a U.S. National Science Foundation Graduate Fellowship. 


\section{References}

1. Zvara, I., Eichler, B., Belov, V. Z., Zvarova, T. S., Korotkin, Yu. S., Shalaevskii, M. R., Shchegolev, V. A., Hussonnois, M.: Sov. Radiochem. 16, 709 (1974).

2. Zvara, I., Belov, V. Z., Domanov, V. P., Shalaevskii, M. R.: Sov. Radiochem. 18, 328 (1976).

3. Gregorich, K. E., Henderson, R. A., Lee, D. M., Nurmia, M. J., Chasteler, R. M., Hall, H. L., Bennett, D. A., Gannett, C. M., Chadwick, R. B., Leyba, J. D., Hoffman, D. C., Herrmann, G.: Radiochim. Acta 43, 223 (1988).

4. Keller, O. L., Seaborg, G. T.: Ann. Rev. Nucl. Sci. 27, 139 (1977).

5. Hyde, E. K., Hoffman, D. C., Keller, O. L.: Radiochim. Acta 42, 57 (1987).

6. Stevenson, P. C., Hicks, H. G.: Anal. Chem. 25, 1517 (1953).

7. Werning, J. R., Higbie, K. B., Grace, J. T., Speece, B. F., Gilbert, H. L.: Ind. Eng. Chem. 46, 644 (1954).

8. Milner, G. W. C., Barnett, G. A., Smales, A. A.: Analyst 80, $380(1955)$

9. Desclaux, J.-P.: At. Data Nucl. Data Tables 12, 311 (1973).

10. Schädel, M., Brüchle, W., Jäger, E., Schimpf, E., Kratz, J. V., Scherer, U. W., Zimmermann, H. P.: Radiochim. Acta 48, $171-176$ (1989).

11. Ghiorso, A., Nurmia, M., Eskola, K., Eskola, P.: Phys. Rev. C4, 1850 (1971)

12. Bemis, C. E., Ferguson, R. L., Plasil, F., Silva, R. J., O’Kelley, G. D., Hahn, R. L., Keifer, M. L., Hensley, D. C.: Phys. Rev. Lett. 39, 1246 (1977).

13. Bemis, C. E., Ferguson, R. L., Plasil, F., Silva, R. J., O'Kelley, G. D., Hahn, R. L., Hensley, D. C., Hulet, E. K., Lougheed, R. W.: ORNL Chemistry Division Annual Progress Report, ORNL-5927, p. 85 (1977).

14. Druin, V. A., Bochev, B., Lobanov, Yu. V., Sagaidak, R. N., Kharitonov, Yu. P., Tret'yakova, S. P., Gul'bekyan, G. G., Buklanov, G. V., Erin, E. A., Kosyakov, V. N., Rykov, A. G.: Sov. J. Nucl. Phys. 29, 591 (1979).

15. Gregorich, K. E., unpublished data (1986).
16. Ellis-Akovali, Y. A., Harmatz, B., Martin, M. J., Ewbank, W. B., Schmorak, M. R., Ball, S. J., McGinnis, M. R., Miller, J. T., Hurley, F. W., Smith, J. E.: Nuclear Data Sheets Vol. 32, New York: Academic Press, 1981, p. 203.

17. Zimmermann, H. P.: Diploma thesis, Universität Mainz (1988).

18. Data of Inorganic Solvent Extraction, Ishimori, T., Nakamura, E., eds., Japan Atomic Energy Research Institute Research Report JAERI 1047 (1963).

19. Markl, P.: Extraktion und Extraktionschromatographie in der Anorganischen Chemie, Methoden der Analyse in der Chemie, Vol. 13, Akademische Verlagsgesellschaft, Frankfurt (1972).

20. Brinkmann, A. Th., de Vries, G., van Dalen, E.: J. Chromatogr. 31, 182 (1967).

21. Zolokazov, V. B.: Nucl. Instrum. Methods 151, 303 (1978).

22. Hoffmann, D. C.: Acc. Chem. Res. 17, 235 (1984), and Hulet, E. K., Wild, J. F., Dougan, R. J., Lougheed, R. W., Landrum, J. H., Dougan, A. D., Schädel, M., Hahn, R. L., Baisden, P. A., Henderson, C. M., Dupzyk, R. J., Sümmerer, K., Bethune, G. R.: Phys. Rev. Lett. 56, 313 (1986).

23. Comprehensive Coordination Chemistry, ed. Wilkinson, G., Vol. 3, p. 591, Pergamon (1987)

24. Ellenburg, J. Y., Leddicotte, G. W., Moore, F. L.: Anal. Chem. 26, 1045 (1954).

25. Leddicotte, G. W., Moore, F. L.: J. Am. Chem. Soc. 74, 59 (1952).

26. Solvent Extraction Reviews, ed. Marcus, Y., Vol. 1, p. 133, Marcel Dekker, New York (1971).

27. For the group-VB elements these are 1.10 atomic units $(\mathrm{Nb})$, 1.14 au for Ta, and 1.29 au for Ha, Fricke, B., Johnson, E., private communication (1989).

28. Pyykkö, P.: Chem. Rev. 88, 563 (1988).

29. Pyykkö, P., Desclaux, J.-P.: Acc. Chem. Res. 12, 276 (1979). 
